\title{
Comparative evaluation of amniotic fluid as an alternative to fetal bovine serum in the maintenance of Leishmania major and Toxoplasma gondii
}

\author{
Zahra Rahmani $^{1} \cdot$ Roghiyeh Faridnia $^{2} \cdot$ Hamed Kalani $^{3}$ (D) Naryan Ghanei ${ }^{2} \cdot$ Mahdi Fakhar $^{4,5} \cdot$ Marzieh Zamanian $^{1,4}$. \\ Masoud Keighobadi ${ }^{4} \cdot$ Rabeeh Tabaripour $^{5}$
}

Received: 26 June 2020 / Accepted: 17 January 2021 / Published online: 25 January 2021

(C) The Author(s), under exclusive licence to Springer-Verlag GmbH, DE part of Springer Nature 2021

\begin{abstract}
This study aimed to evaluate the efficacy of amniotic fluid (AF) as an alternative to fetal bovine serum (FBS) in the maintenance of Leishmania major promastigotes and Toxoplasma gondii tachyzoites. AF was collected by an obstetrician using sterile syringes during a cesarean section. The culture medium was supplemented with 5 different concentrations of FBS or AF including 2, 5, 10,20, and 30\%. These concentrations were used to maintain both mentioned parasites. L. major was maintained at temperatures 4 and $24{ }^{\circ} \mathrm{C}$ and examined once a week for 4 weeks, while $T$. gondii was maintained at temperatures 4,24 , and 37 ${ }^{\circ} \mathrm{C}$ and examined at hours $24,48,72$, and 96 . For $L$. major, at both 4 and $24{ }^{\circ} \mathrm{C}$, we observed no significant difference between FBS and AF on day 7. However, on days 14, 21, and 28, the difference between FBS and AF was significant at both temperatures. For $T$. gondii, no significant difference was observed between FBS and AF at hour 24 and all temperatures. However, this difference was significant at hours 48, 72, and 96 and all temperatures. According to our results, although FBS had a greater efficacy than $\mathrm{AF}$ in the growth of $L$. major and the survival of $T$. gondii, the number of promastigotes increased over time in AFcontaining medium and the number of tachyzoites reduced slowly with a mild slop. Therefore, AF can be a potential alternative to FBS.
\end{abstract}

Keywords Amniotic fluid $\cdot$ Culture $\cdot$ Fetal bovine serum $\cdot$ Leishmania major $\cdot$ Toxoplasma gondii

\section{Introduction}

Leishmania major (L. major) and Toxoplasma gondii (T. gondii) are parasitic protozoa, which infect human (Walker et al. 2014). These parasites have always been an interesting issue for many researchers. Therefore, maintenance

\begin{tabular}{l}
\hline Section Editor: Nawal Hijjawi \\
\hline$\square \quad \begin{array}{l}\text { Roghiyeh Faridnia } \\
\text { r_faridnia400@yahoo.com }\end{array}$ \\
$\square \quad$ Hamed Kalani \\
hamed.kalani@yahoo.com \\
$\square \quad$ Mahdi Fakhar \\
mahdif53@yahoo.com \\
$1 \quad$ Department of Obstetrics and Gynecology, Imam Khomeini \\
Hospital, Mazandaran University of Medical Sciences, Sari, Iran \\
Student Research Committee, Mazandaran University of Medical \\
Sciences, Sari, Iran
\end{tabular}

of these parasites in the laboratory is essential and important for research.

The parasite cultivation techniques are used to isolate parasites from clinical samples for diagnosis and to study their various aspects such as physiological, biochemical, and antigenic features. In addition, cultivation has numerous values in

3 Infectious Diseases Research Center, Golestan University of Medical Sciences, Gorgan, Iran

4 Toxoplasmosis Research Center, Communicable Diseases Institute, Iranian National Registry Center for Toxoplasmosis (INRCT), Mazandaran University of Medical Sciences, Sari, Iran

5 Toxoplasmosis Research Center, Communicable Diseases Institute, Iranian National Registry Center for Lophomoniasis (INRCL), Imam Khomeini Hospital, Mazandaran University of Medical Sciences, Sari, Iran 
vaccine design and evaluation of its effectiveness, specific antigen extraction, drug-resistance assessment, and screening of potential therapeutic agents (Ahmed 2014; Visvesvara and Garcia 2002).

RPMI-1640, one of the basic liquid culture media, is a growth medium used in cell culture. Fetal bovine serum (FBS) with a final concentration of $10-30 \%$ is commonly used as a supplementary medium for better cell growth and to enrich the cell and tissue culture media (Helgason and Miller 2005; Limoncu et al. 2004). This culture medium contains many growth-supporting compounds, nutrients, and energy sources, and it is the most important source of proteins (Ali et al. 1998; Sandle 2014). The main disadvantages of FBS include high cost and toxic effects on cells when used in high amounts in the culture media (Becker et al. 1996; Newman 2003).

Amniotic fluid (AF) surrounds the embryo and fetus during growth. AF has many functions, and it protects the fetus from infectious agents due to innate antibacterial properties. Moreover, it serves as a reservoir of fluid and nutrients, containing proteins, electrolytes, immunoglobulins, and vitamins (Tong et al. 2009). During childbirth, the amniotic sac ruptures and the fluid inside it comes out. Therefore, AF can be easily collected and is available for free (Kamath-Rayne et al. 2014), and these are the reasons why we focused on it.

Accordingly, in the present study, for the first time, AF was used as an alternative to FBS in the growth and maintenance of L. major and T. gondii.

\section{Materials and methods}

\section{Amniotic fluid collection}

In this experimental study, AFs were collected by an obstetrician using sterile syringes during a cesarean section at the Imam Khomeini Hospital, Sari, Iran. The AF was transferred to the parasitology laboratory of the Mazandaran University of Medical Sciences (MAZUMS), Sari, Iran, and after centrifuging at $3000 \times g$ for $10 \mathrm{~min}$, the supernatant was filter-sterilized using a $0.22-\mu \mathrm{m}$ pore size syringe filter (Corning, Oneonta, NY, USA) and stored at $-20{ }^{\circ} \mathrm{C}$ until used. This study was reviewed and approved by the
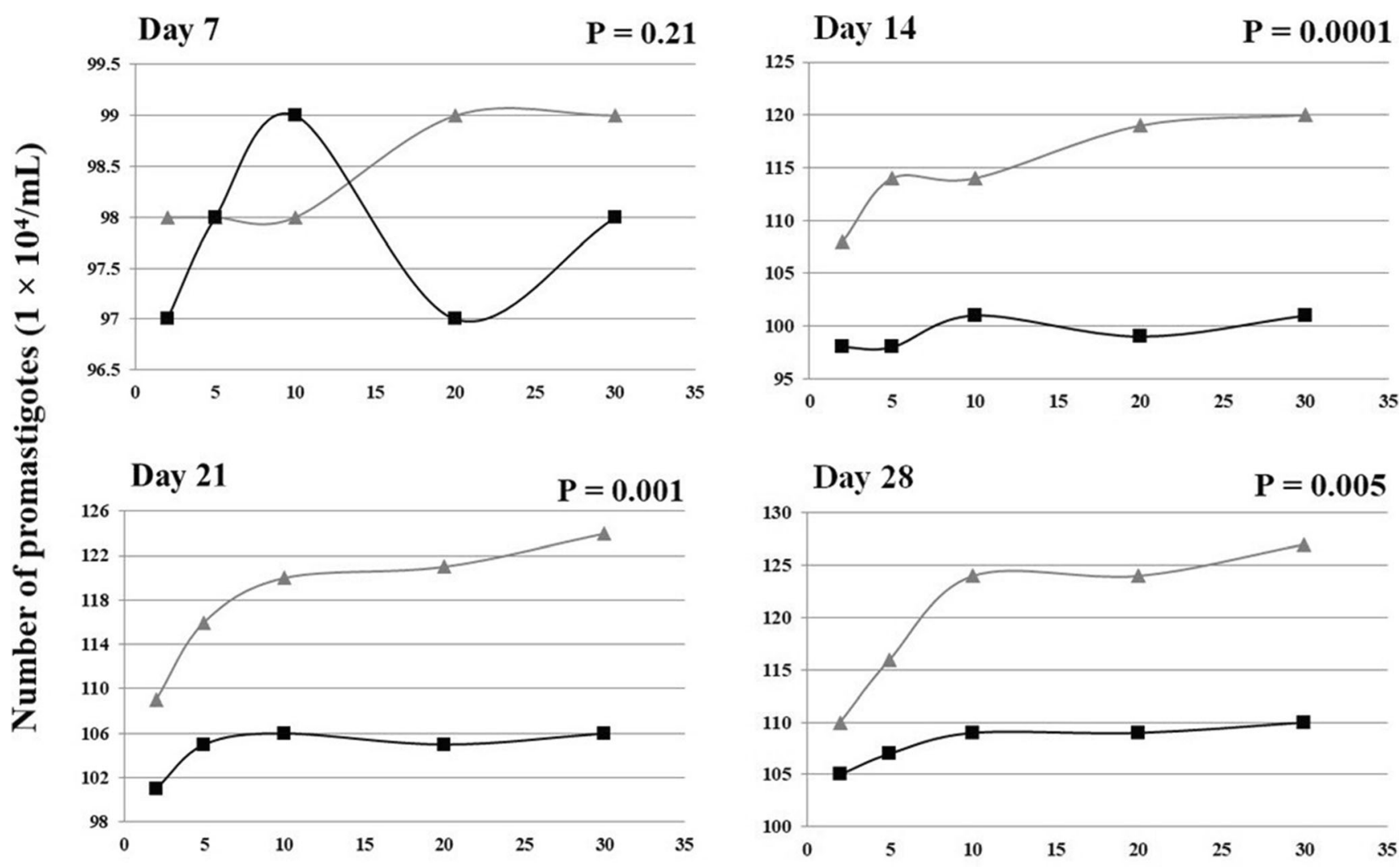

Concentrations (\%)

Fig. 1 The number of promastigotes in both media, fetal bovine serum (FBS) and amniotic fluid (AF), at $4{ }^{\circ} \mathrm{C}$ in different days. The number of promastigotes was calculated using a hemocytometer under a light microscope with $\times 400$ magnification. Triangle, FBS; square, AF 
Research Ethics Committee of MAZUMS (Ethical Code: IR.MAZUMS.REC.1399.361). An informed consent was obtained from all participants.

\section{Assessment of AF or FBS on L. major maintenance}

For this purpose, L. major promastigotes (MRHO/IR/75/ ER), maintained in laboratory conditions, were used (Faridnia et al. 2018). RPMI-1640 medium (Gibco, Grand Island, NY, USA) was supplemented with 5 different concentrations of FBS (Gibco, Grand Island, NY, USA) or AF including 2, 5, 10, 20, and $30 \%$ in a volume of $4.5 \mathrm{~mL}$ each. Two sets of these 5 concentrations were prepared for each of FBS or AF to which $2.5 \times 10^{6} \mathrm{~L}$. major promastigotes in a volume of 0.5 $\mathrm{mL}$ was added. The final volume was $5 \mathrm{~mL}$ for each tube. The total number of tubes was 20 , of which 10 tubes were considered for FBS and 10 other ones for $\mathrm{AF}$. Out of 10 tubes for each of FBS or AF, 5 were incubated at $4{ }^{\circ} \mathrm{C}$ and 5 other ones at $24{ }^{\circ} \mathrm{C}$. The tubes were examined once a week for 4 weeks. Accordingly,
Table 1 Comparison of the number of promastigotes in FBS and AF during times ( 4 weeks) and at each of 4 and $24{ }^{\circ} \mathrm{C}$ by repeated-measures ANOVA

\begin{tabular}{rrrrr}
\hline Medium & Degree centigrade & \multicolumn{1}{c}{$\mathrm{F}^{\mathrm{a}}$} & $\mathrm{df}^{\mathrm{b}}$ & $p$ value \\
\hline FBS $^{\mathrm{c}}$ & 4 & 53.1 & 3 & $<0.00001$ \\
& 24 & 30.79 & 3 & $<0.00001$ \\
\multirow{2}{*}{$\mathrm{AF}^{\mathrm{d}}$} & 4 & 91.5 & 3 & $<0.00001$ \\
& 24 & 50.4 & 3 & $<0.00001$ \\
\hline
\end{tabular}

${ }^{\mathrm{a}}$ F-table

${ }^{\mathrm{b}}$ Degrees of freedom

${ }^{\mathrm{c}}$ Fetal bovine serum

${ }^{\mathrm{d}}$ Amniotic fluid

$20 \mu \mathrm{L}$ of each tube contents was added to $20 \mu \mathrm{L}$ of $2 \%$ formaldehyde in phosphate-buffered saline (PBS; $\mathrm{pH}=$ 7.2). The number of promastigotes was then counted using a hemocytometer under a light microscope with $\times 400$ magnification. The examination was performed in triplicate for each tube.

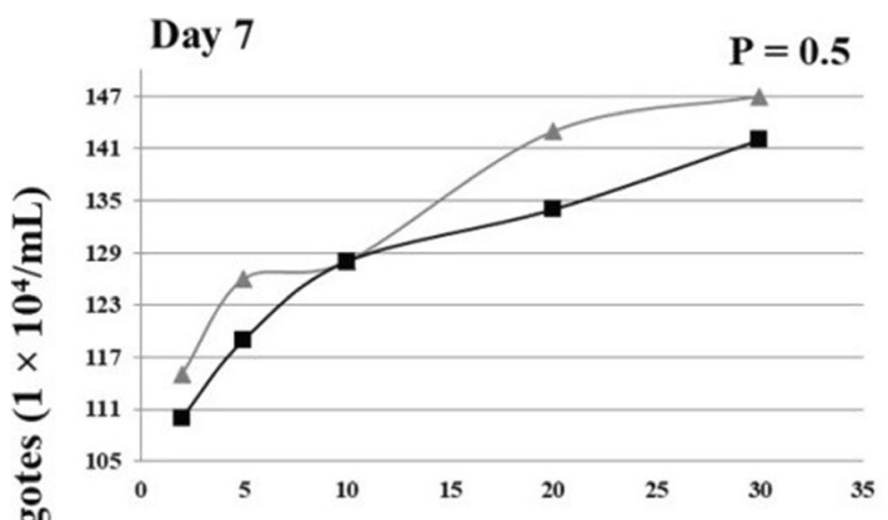

Day 21

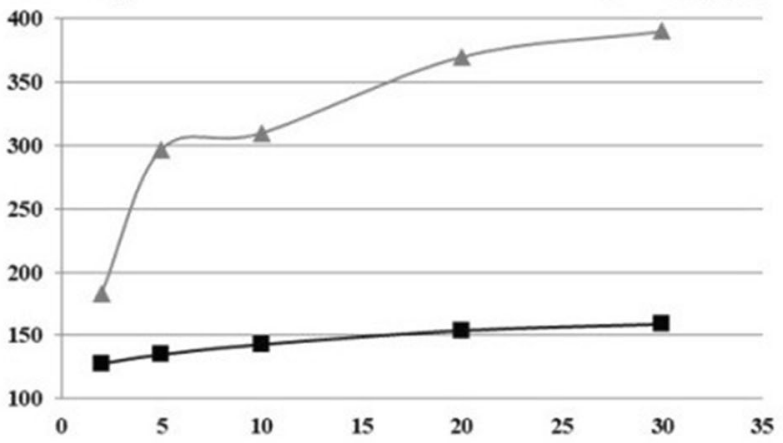

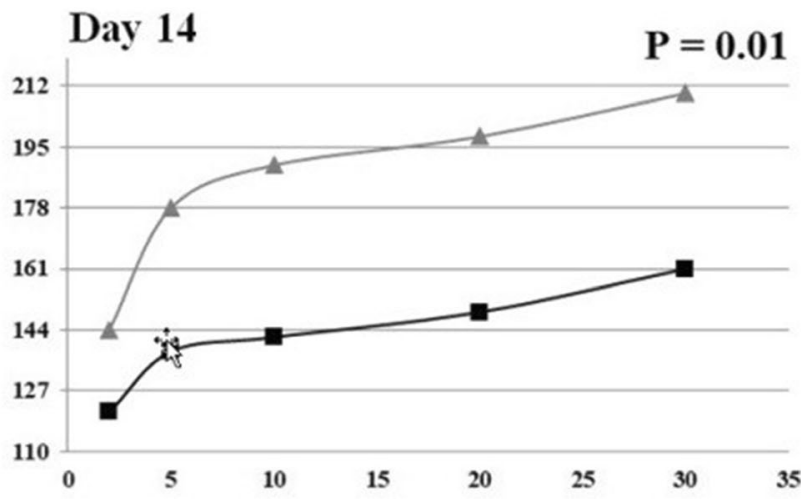

Day 28

$\mathrm{P}=\mathbf{0 . 0 0 0 3}$

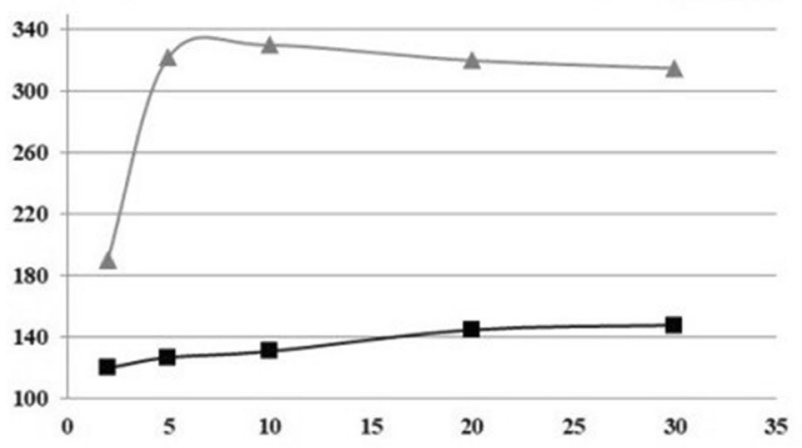

\section{Concentrations (\%)}

Fig. 2 The number of promastigotes in both media, fetal bovine serum (FBS) and amniotic fluid (AF), at $24{ }^{\circ} \mathrm{C}$ in different days. The number of promastigotes was calculated using a hemocytometer under a light microscope with $\times 400$ magnification. Triangle, FBS; square, AF 


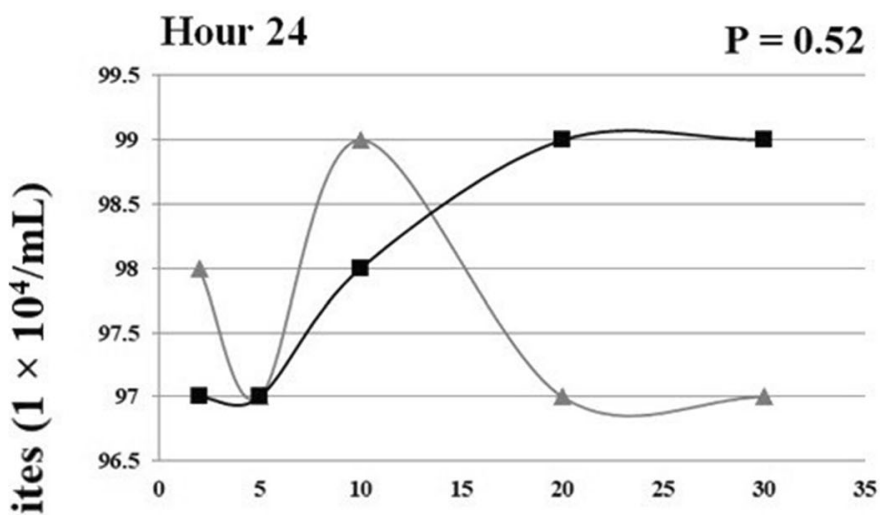

\section{Hour 48}

$\mathbf{P}=\mathbf{0 . 0 0 0 3}$

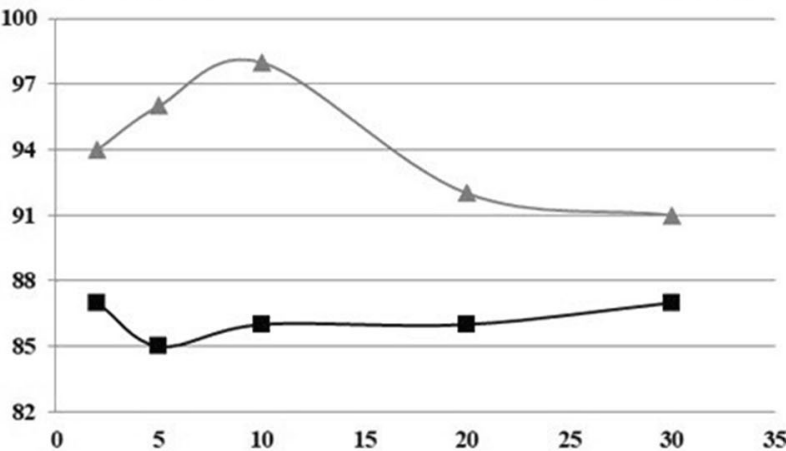

Hour 72

$\mathbf{P}=\mathbf{0 . 0 0 0 0 1}$

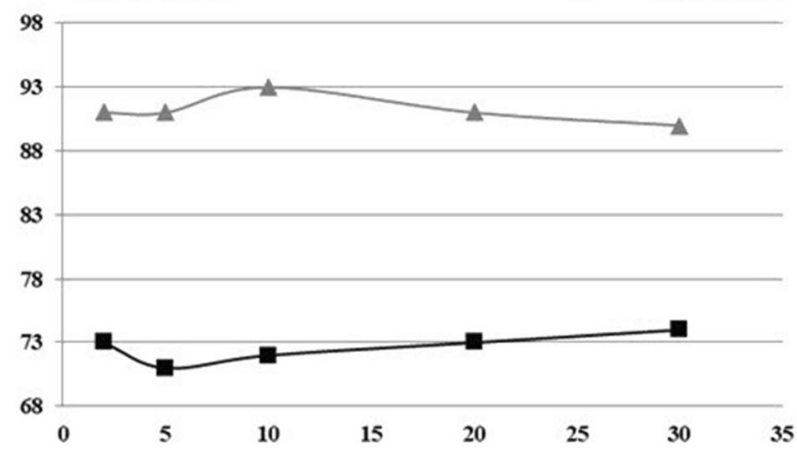

Hour 96

$\mathbf{P}=\mathbf{0 . 0 0 0 0 1}$

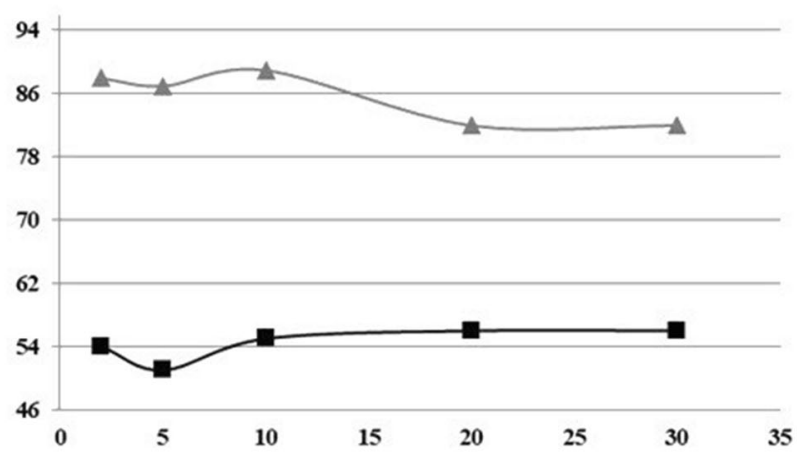

\section{Concentration (\%)}

Fig. 3 The number of tachyzoites in both media, fetal bovine serum (FBS) and amniotic fluid (AF), at $4{ }^{\circ} \mathrm{C}$ in different days. The number of tachyzoites was calculated using a hemocytometer under a light microscope with $\times 400$ magnification. Triangle, $\mathrm{FBS}$; square, $\mathrm{AF}$

\section{Assessment of AF or FBS on T. gondii maintenance}

Freshly harvested tachyzoites of $T$. gondii $\mathrm{RH}$ strain from cell culture medium were used for this purpose (Kalani et al. 2016b). RPMI-1640 medium was supplemented with 5 different concentrations of FBS or AF including 2, 5, 10, 20, and $30 \%$ in a volume of $4.5 \mathrm{~mL}$ each. Three sets of these 5 concentrations were prepared for each of FBS or AF to which $5 \times$ $10^{6} \mathrm{~T}$. gondii tachyzoites in a volume of $0.5 \mathrm{~mL}$ was added. The final volume was $5 \mathrm{~mL}$ for each tube. The total number of tubes was 30, of which 15 tubes were considered for FBS and 15 other ones for AF. Out of 15 tubes for each of FBS or AF, 5 were incubated at $4{ }^{\circ} \mathrm{C}, 5$ at $24^{\circ} \mathrm{C}$, and 5 other ones at $37^{\circ} \mathrm{C}$. The tubes were examined at hours 48, 72, and 96. Accordingly, the number of tachyzoites was counted in 20 $\mu \mathrm{L}$ of each tube contents using a hemocytometer under a light microscope with $\times 400$ magnification. The examination was performed in triplicate for each tube.

\section{Statistical analysis}

The IBM SPSS v16 software (IBM SPSS Inc., Chicago, IL, USA) was utilized for statistical analysis. The repeated measures analysis of variance (ANOVA) and two-tailed $t$ test was used for analysis and the results were significant where $p$ value was $<0.05$.

\section{Results}

\section{L. major maintenance in AF and FBS media}

The results showed that the number of promastigotes at $4{ }^{\circ} \mathrm{C}$ on day 7 was not significantly different between the FBS and AF groups $(p=0.21)$. However, on days 14,21 , and 28 , this difference was significant and the highest difference was observed on day 14 ( $p=0.0001)$ (Fig. 1). Moreover, at $24^{\circ} \mathrm{C}$ on day 7 , there was not a significant difference between these two groups $(p=0.5)$. However, on days 14,21 , and 28, the difference was significant and the highest difference was observed on day 28 ( $p=0.0003$ ) (Fig. 2).

The results of comparing the number of promastigotes in each of the two media (FBS and AF) separately at different times and temperatures 4 and $24{ }^{\circ} \mathrm{C}$ showed that there was a significant difference in the number of promastigotes $(p<$ 0.05) (Table 1). 


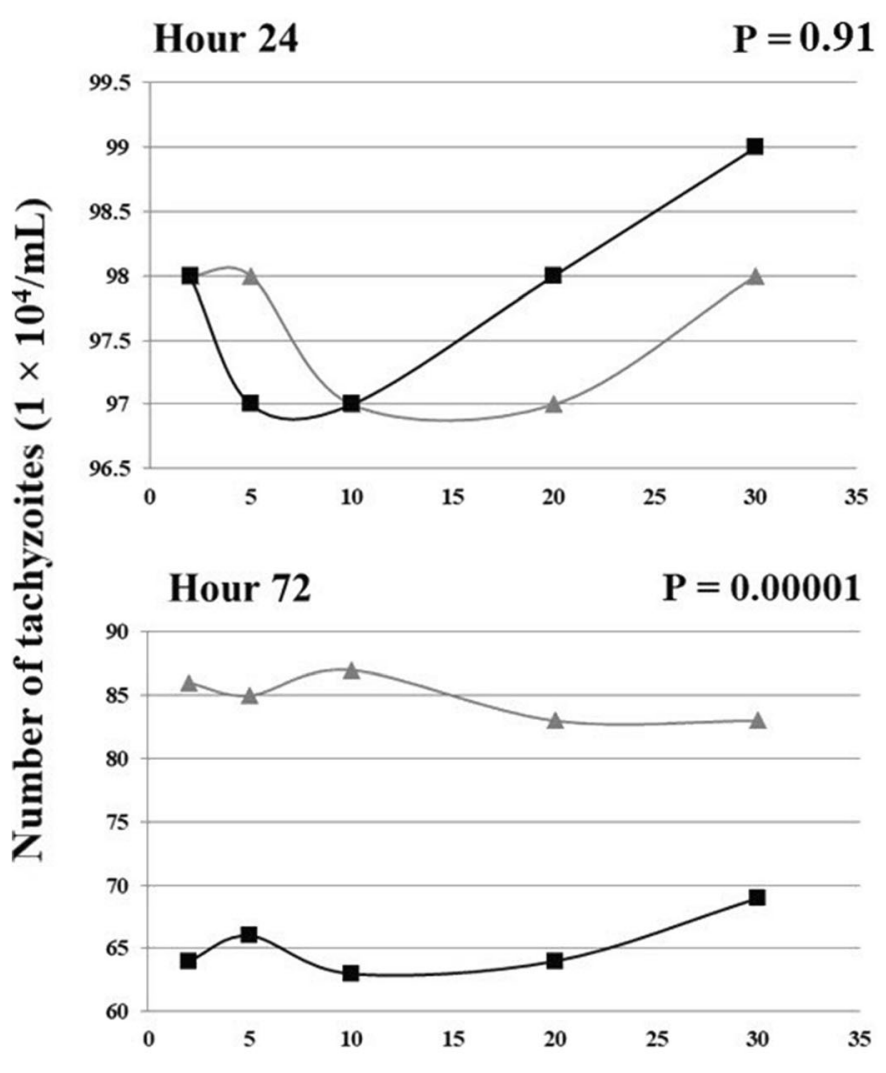

Hour 48

$\mathbf{P}=0.00001$

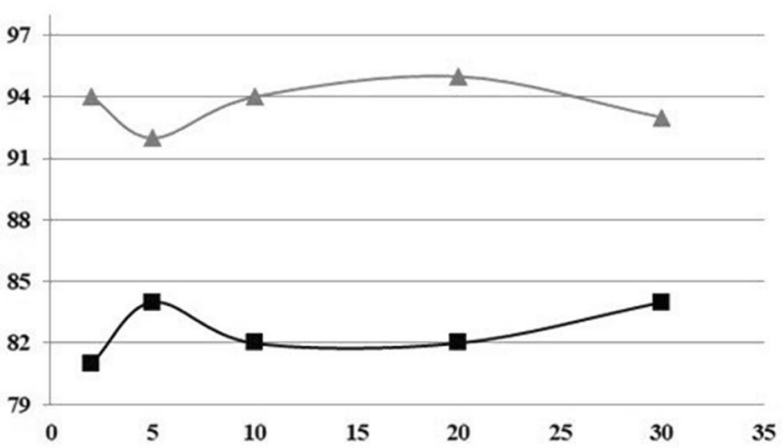

Hour 96

$\mathbf{P}=\mathbf{0 . 0 0 0 1}$

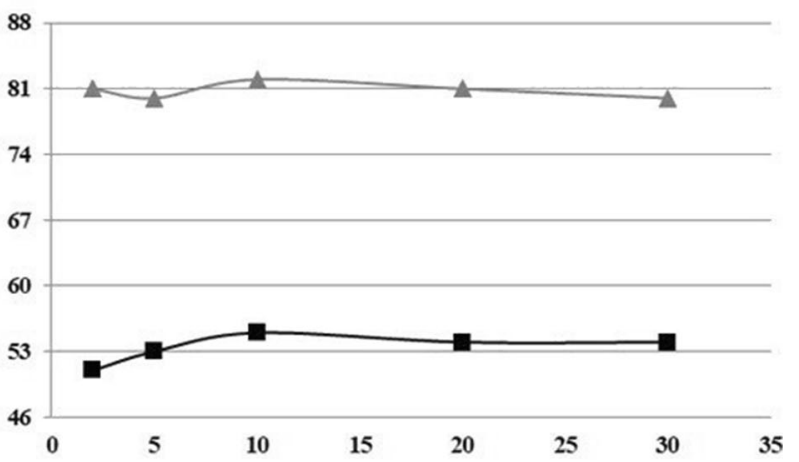

Concentration (\%)

Fig. 4 The number of tachyzoites in both media, fetal bovine serum (FBS) and amniotic fluid (AF), at $24^{\circ} \mathrm{C}$ in different days. The number of tachyzoites was calculated using a hemocytometer under a light microscope with $\times 400$ magnification. Triangle, FBS; square, $\mathrm{AF}$

\section{T. gondii maintenance in AF and FBS media}

The results showed that the number of tachyzoites at $4{ }^{\circ} \mathrm{C}$ and hour 24 was not significantly different between the FBS and AF groups $(p=0.52)$. However, at hours 48,72 , and 96 , this difference was significant and the highest difference was observed at hours 72 and $96(p=$ $0.00001)$ (Fig. 3). Furthermore, at hour 24 and temperatures of $24{ }^{\circ} \mathrm{C}(p=0.91)$ and $37{ }^{\circ} \mathrm{C}(p=0.37)$, there was not a significant difference between these two groups (Figs. 4 and 5). However, at hours 48, 72, and 96, this difference was significant and the highest difference was observed at hours 48 and 72 ( $p=0.00001)$ (Fig. 4). At 37 ${ }^{\circ} \mathrm{C}$ and hour 24 , no significant difference was observed between the FBS and AF groups $(p=0.37)$. However, at hours 48,72 , and 96 , this difference was significant and the highest difference was observed at hour $72(p=$ 0.00001) (Fig. 5).

The results of comparing the number of tachyzoites in each of the two media (FBS and AF) separately at different times and temperatures 4,24 , and $37{ }^{\circ} \mathrm{C}$ showed that there was a significant difference in the number of tachyzoites $(p<0.05)$ (Table 2).

\section{Discussion}

In vitro techniques are important for cell and tissue culture. The most frequently used supplementary solution in the media is FBS, containing hormones, vitamins, salts, electrolytes, and other growth factors that should be free of blood cells, platelets, or other coagulation factors (Jochems et al. 2002).

Studies have showed that growth factors in AF play an important role in the growth and development of the human embryo (Hui and Bianchi 2011). The effect of AF on the growth of human corneal endothelial cells was compared with FBS, and the researchers concluded that these cells proliferated and grew more rapidly in DMEM-F12 supplemented with $20 \%$ AF than those supplemented with $20 \%$ FBS. Furthermore, these cells were appropriately established in DMEM-F12 containing 20\% AF and their morphology was not changed. Therefore, the authors suggested the use of AF instead of FBS in the cell culture medium (Feizi et al. 2014). In the present study, we observed that there was a significant difference in the number of $L$. major promastigotes at both 4 and $24^{\circ} \mathrm{C}$ on each of the days 14,21 , and 28 between AF and FBS groups. Moreover, there was a significant difference in the number of $T$. gondii tachyzoites at 4,24 , and $37^{\circ} \mathrm{C}$ and 
Hour 24

$\mathbf{P}=\mathbf{0 . 3 7}$

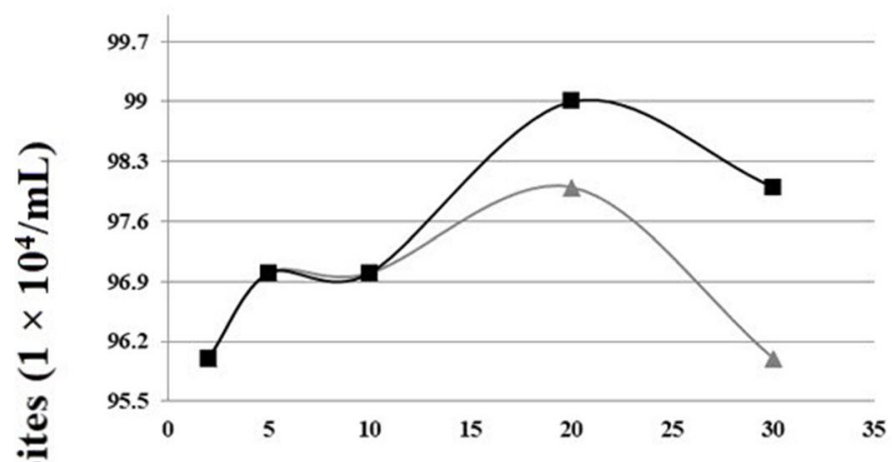

Hour 72
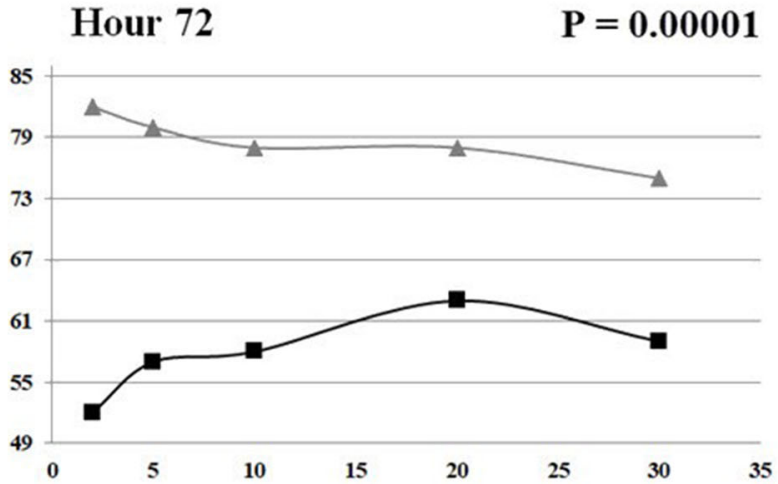

Hour 48

$\mathbf{P}=0.00007$

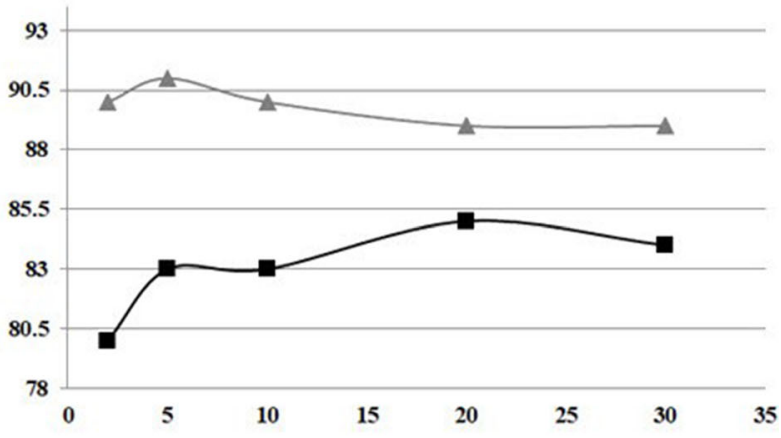

Hour 96

$\mathbf{P}=\mathbf{0 . 0 0 2}$

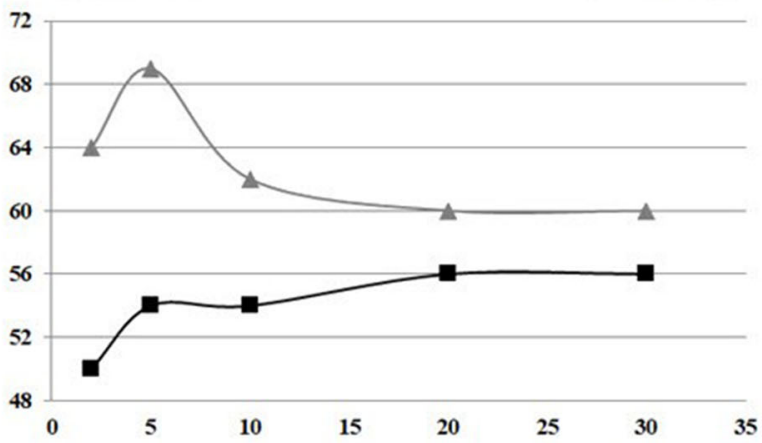

Concentration

Fig. 5 The number of tachyzoites in both media, fetal bovine serum (FBS) and amniotic fluid (AF), at $37^{\circ} \mathrm{C}$ in different days. The number of tachyzoites was calculated using a hemocytometer under a light microscope with $\times 400$ magnification. Triangle, FBS; square, $\mathrm{AF}$

each of 48, 72, and $96 \mathrm{~h}$ between AF and FBS groups. Therefore, the effect of FBS was better in the maintenance of both the parasites than AF. It should also be noted that the latter study examined the effect of AF on corneal endothelial cells, but we did it on the two parasites.

The survival rate and viability of $T$. gondii tachyzoites in cow milk and ovine hydatid cyst fluid (HCF) in comparison

Table 2 Comparison of the number of tachyzoites in FBS and AF during times $(96 \mathrm{~h})$ and at each of 4,24 , and $37^{\circ} \mathrm{C}$ by repeatedmeasures ANOVA

\begin{tabular}{crrrr}
\hline Medium & Degree centigrade & \multicolumn{1}{c}{$\mathrm{F}^{\mathrm{a}}$} & $\mathrm{df}^{\mathrm{b}}$ & \multicolumn{1}{c}{$p$ value } \\
\hline FBS $^{\mathrm{c}}$ & 4 & 22.29 & 3 & 0.00003 \\
& 24 & 220.6 & 3 & $<0.00001$ \\
& 37 & 255.3 & 3 & $<0.00001$ \\
$\mathrm{AF}^{\mathrm{d}}$ & 4 & 957.4 & 3 & $<0.00001$ \\
& 24 & 911.9 & 3 & $<0.00001$ \\
& 37 & 1095.5 & 3 & $<0.00001$ \\
\hline
\end{tabular}

${ }^{\mathrm{a}} \mathrm{F}$-table

${ }^{\mathrm{b}}$ Degrees of freedom

${ }^{\mathrm{c}}$ Fetal bovine serum

${ }^{\mathrm{d}}$ Amniotic fluid with RPMI-1640 supplemented with $10 \%$ and $20 \%$ FBS showed that tachyzoites were more notably survived in ovine HCF than the medium with $20 \%$ FBS. Survival time in milk and FBS-supplemented media was almost the same and there was no significant difference. The authors in the recent study concluded that the best condition for $T$. gondii tachyzoite maintenance was PBS at $4{ }^{\circ} \mathrm{C}$, in which the parasite survived for 12 days (Kalani et al. 2016a). However, this finding needs further evaluation since other researchers have showed that RPMI-1640 is more successful than PBS in the maintenance of $T$. gondii tachyzoites (El-Bahy et al. 2018). In the present study, we observed that the FBS-supplemented medium is more appropriate than AF for $T$. gondii tachyzoite maintenance.

In another study, L. major promastigotes were maintained in different solutions, including brain heart infusion (BHI) broth, BHI with $5 \%$ FBS, BHI with $10 \% \mathrm{FBS}, \mathrm{HCF}, \mathrm{BHI}$ with $5 \% \mathrm{HCF}$, and $\mathrm{BHI}$ with $10 \% \mathrm{HCF}$. The findings indicated that promastigotes were notably proliferated in $\mathrm{BHI}$ with $10 \%$ FBS as compared to other media, in which the highest number of promastigotes $\left(448 \times 10^{5}\right)$ was observed on day 6 and at $24{ }^{\circ} \mathrm{C}$. This value at this time was $96 \times 10^{5}$ for $\mathrm{HCF}$, $224 \times 10^{5}$ for BHI with $5 \% \mathrm{HCF}$, and $512 \times 10^{5}$ for BHI with $10 \% \mathrm{HCF}$, indicating a difference significantly between $\mathrm{BHI}$ 
with $10 \%$ FBS and HCF alone (Fakhar et al. 2006). However, we observed that there was no significant difference in the number of promastigotes between AF and FBS media on day 7 at $24^{\circ} \mathrm{C}(p=0.5)$, suggesting that $\mathrm{AF}$ can be considered an alternative for FBS in the short-term. Most importantly, the number of promastigotes increased over time (days 7 to 28) in $\mathrm{AF}$-containing medium, indicating that $\mathrm{AF}$ is even a reliable alternative for FBS for long-term period.

Researchers in different studies used various rich solutions for a possible alternative to FBS. In a study, the efficacy of 10\% goat, cow, and buffalo milk in medium 199 was examined and compared to medium 199 with $10 \%$ FBS for the proliferation of Leishmania donovani promastigotes. The results showed that the number of promastigotes was not statistically different between the medium supplemented with FBS and the media containing various types of milk, indicating that milk can be used as an appropriate alternative to FBS (Muniaraj et al. 2007).

\section{Conclusion}

Our results showed that FBS has a greater efficacy than AF in the growth of $L$. major and the survival of $T$. gondii. However, the number of $L$. major promastigotes increased over time in AF-containing medium; therefore, $\mathrm{AF}$ can be a potential alternative to FBS. Although FBS was much better than AF in $T$. gondii maintenance, the number of tachyzoites reduced slowly with a mild slop in AF-containing medium as compared to FBS. Accordingly, further studies are recommended on AF and its efficacy on the maintenance of the protozoan parasites as an inexpensive, reliable, and available supplementary solution instead of FBS.

Acknowledgements The authors would like to thank the participants for their contribution in this study. We are very grateful to Dr. Masoud Keighobadi for all his efforts. He did not response to treatment and expired on January 21, 2021, due to COVID-19. God rest his soul.

Funding This study was supported by the Vice Chancellor of Research at the Mazandaran University of Medical Sciences (Code: 7049).

\section{Compliance with ethical standards}

Conflict of interest The authors declare no competing interests.

\section{References}

Ahmed NH (2014) Cultivation of parasites. Tropical parasitology 4:8089

Ali SA, Iqbal J, Ahmad B, Masoom M (1998) A semisynthetic fetal calf serum-free liquid medium for in vitro cultivation of Leishmania promastigotes. The American Journal of Tropical Medicine and Hygiene 59:163-165
Barker RDJ (1999) Biotechnology: A laboratory course (2th ed) by Becker JM, Caldwell GA, and Zachgo EA. pp 261. Academic Press, San Diego, 1996.

El-Bahy MM, Khalifa MM, Méabed EM (2018) Toxoplasma gondii: prolonged in-vitro maintenance of virulent tachyzoites in fluid media at low temperatures. Alexandria Journal of Medicine 54:511515

Fakhar M, Habibi P, Motazedian M (2006) Evaluation of hydatid cyst fluid as a substitute for fetal bovine serum (FRS) in culture of leishmania major. Zahedan Journal of Research in Medical Sciences 8: $47-52$

Faridnia R, Kalani H, Fakhar M, Akhtari J (2018) Investigating in vitro anti-leishmanial effects of silibinin and silymarin on Leishmania major. Annals of parasitology 64:29-35

Feizi S, Soheili ZS, Bagheri A, Balagholi S, Mohammadian A, RezaeiKanavi M, Ahmadieh H, Samiei S, Negahban K (2014) Effect of amniotic fluid on the in vitro culture of human corneal endothelial cells. Experimental eye research 122:132-140

Helgason CD, Miller CL (2005) Basic cell culture protocols. Totowa, NJ: Humana Press.

Hui L, Bianchi D (2011) Cell-free fetal nucleic acids in amniotic fluid. Human reproduction update 17:362-371

Jochems CE, Van Der Valk JB, Stafleu FR, Baumans V (2002) The use of fetal bovine serum: ethical or scientific problem? Alternatives to Laboratory Animals 30:219-227

Kalani H et al (2016a) Comparison of eight cell-free media for maintenance of Toxoplasma gondii tachyzoites. Iranian journal of parasitology 11:104-109

Kalani H, Shahreza HK, Daryani A, Yousefi HA, Pestechian N, Mansouri V (2016b) The gene expression level of IFN- $\gamma$ R1 and IFN- $\gamma \mathrm{R} 2$ in a murine model treated with Toxoplasma gondii and its products. Gastroenterology and hepatology from bed to bench 9: 124-131

Kamath-Rayne BD, Smith HC, Muglia LJ, Morrow AL (2014) Amniotic fluid: the use of high-dimensional biology to understand fetal wellbeing. Reproductive Sciences 21:6-19

Limoncu M, Özbilgin A, Balcioḡlu İ, Özbel Y (2004) Evaluation of three new culture media for the cultivation and isolation of Leishmania parasites. Journal of Basic Microbiology: An International Journal on Biochemistry, Physiology, Genetics, Morphology, and Ecology of Microorganisms 44:197-202

Muniaraj M, Lal C, Kumar S, Sinha P, Das P (2007) Milk of cow (Bos taurus), buffalo (Bubalus bubalis), and goat (Capra hircus): a better alternative than fetal bovine serum in media for primary isolation, in vitro cultivation, and maintenance of Leishmania donovani promastigotes. Journal of clinical microbiology 45:1353-1356

Newman C (2003) Serum-Free Cell Culture Biomedical scientist 47:941943

Sandle T (2014) Assessment of culture media in pharmaceutical microbiology American Pharmaceutical Review[En línea] Disponible en: $\mathrm{http}: / / \mathrm{www}$ americanpharmaceuticalreview com/Featured-Articles/ 163589-Assessmentof-Culture-Media-in-PharmaceuticalMicrobiology/Recopilado 15:2017

Tong X-L, Wang L, Gao T-B, Qin Y-G, Qi Y-Q, Xu Y-P (2009) Potential function of amniotic fluid in fetal development — novel insights by comparing the composition of human amniotic fluid with umbilical cord and maternal serum at mid and late gestation. Journal of the Chinese Medical Association 72:368-373

Visvesvara GS, Garcia LS (2002) Culture of protozoan parasites. Clinical Microbiology Reviews 15:327-328

Walker DM, Oghumu S, Gupta G, McGwire BS, Drew ME, Satoskar AR (2014) Mechanisms of cellular invasion by intracellular parasites. Cellular and Molecular Life Sciences 71:1245-1263 